\title{
Poor Biointegration of Porcine Acellular Dermal Matrix Associated with Unfavorable Gingival Healing: A Report of Three Cases
}

\author{
Wassim Manhal ${ }^{1} \odot$, Ghassan Yared ${ }^{2}$, Roula Tahtouh ${ }^{3}$, George Hilal ${ }^{4}$, Claude Ghorra ${ }^{5}$, Didier Lutomski ${ }^{6}$, Sylvie Changotade ${ }^{7}$, \\ Karim Senni ${ }^{8}$, Ronald Younes ${ }^{9}$
}

\begin{abstract}
Aim: The aim of the present work was to explain the poor biointegration of acellular dermal xenogeneic matrix, leading to an unfavorable gingival healing following a grafting procedure for the treatment of soft tissue deficiencies.

Background: Numerous works have demonstrated the successful use of acellular dermal matrix (ADM) in soft tissue augmentation procedures. However, spare human investigations reported adverse healing outcomes at microscopic level.

Case description: Three patients showing various soft tissue deficiencies (recession, gingival thickening) requiring a gingival augmentation were grafted using an $A D M$ porcine acellular dermal matrices (pADM) as a soft tissue substitute. For this purpose, appropriate soft tissue augmentation surgeries were performed and the grafted PADM was left for proper healing. Biopsies were harvested from two out of the three patients, respectively, at 11 and 27 weeks in order to conduct a histological evaluation of the pADM's doubtful biointegration. Moreover, the ultrastructural analysis of pADM was performed using scanning electron microscopy, and additional histological procedures were used to assess its ability to support human gingival fibroblast cultures. Signs of gingival inflammation persisted several months postoperatively. Histologically, numerous inflammatory cells characterized the grafted site. Indeed, the high number of foreign body giant cell granulomas and the very densified newly formed collagen fibers highlighted a fibrotic process within gingival connective tissue.

The ultrastructural and histological analysis showed that pADM was characterized by very thick and dense collagen bundles demonstrating a nonphysiological collagen network organization. Cell culture experiments showed fibroblasts proliferating on the matrix surface, sparing its deeper part, even though the collagen matrix degradation seemed to occur following a gradient from the pADM surface inward.

Conclusion: The unfavorable clinical results may be caused by the poor colonization of matrix cells and poor angiogenesis leading to the inadequate biointegration of pADM. Hence, the PADM structure in terms of porosity and degradability should be further investigated.

Clinical significance: The present cases highlighted a poor integration of pADM following soft tissue grafting procedures, which was caused by the inadequate ultrastructure of the used pADM. Therefore, despite the utility of such tissue substitutes, their manufacturing improvement could be required to obtain a better biointegration.

Keywords: Acellular dermal matrix, Human gingival fibroblasts, Soft tissue augmentation.

The Journal of Contemporary Dental Practice (2021): 10.5005/jp-journals-10024-3167
\end{abstract}

\section{BACKGROUND}

Soft tissue thickness is an important factor in the protection of both natural dentition and dental implants. ${ }^{1}$ Teeth with soft tissue deficiencies cause substantial therapeutic problems, mainly pertaining to aesthetics and hypersensitivity; ${ }^{2}$ on the other hand, a thick peri-implant gingiva plays a major role in reducing the prevalence of peri-implant diseases. ${ }^{3}$ Similarly, for fixed toothsupported prosthesis with keratinized tissue $\geq 2 \mathrm{~mm}$ connective tissue grafts (CTGs) significantly increase the gingival lining, ${ }^{4}$ thus reducing plaque index, gingival inflammation and attachment loss, as compared to equivalent nongrafted sites. ${ }^{5}$ Moreover, it is well known that tooth extraction is accompanied by volumetric tissue changes, more than $60 \%$ of which occur in the first 3 months, thus creating a concavity on the buccal side. ${ }^{6}$

In order to treat such tissue defects, both connective or epithelial-CTGs are regarded nowadays as the gold standard. ${ }^{7}$ Over the years, various modifications to the original technique have been developed, but despite their highly predictable results, they still present numerous disadvantages, particularly an additional surgical site. ${ }^{8}$ To avoid these drawbacks, various alternative solutions have been advocated in the last 10 years, ${ }^{9}$ such as xenogeneic and
1,2 Faculty of Dental Medicine, Division of Oral Biology, Craniofacial Research Laboratory, Saint Joseph University of Beirut, Beirut, Lebanon ${ }^{3,4}$ Faculty of Medicine, Cancer and Metabolism Laboratory, Saint Joseph University, Beirut, Lebanon

${ }^{5}$ Pathology Department, Geitawi Hospital, Beirut, Lebanon

6,7Unité de Recherche Biomatériaux Innovants et Interfaces, Université Sorbonne Paris Nord, Bobigny, Paris, France; Université de Paris, Montrouge, Paris, France

${ }^{8}$ Ecole de Biologie Industrielle, Laboratoire EBInnov, Cergy, Paris, France ${ }^{9}$ Faculty of Dental Medicine, Craniofacial Research Laboratory, Saint Joseph University of Beirut, Beirut, Lebanon

Corresponding Author: Wassim Manhal, Faculty of Dental Medicine, Division of Oral Biology, Craniofacial Research Laboratory, Saint Joseph University of Beirut, Beirut, Lebanon, e-mail: wassim.manhal@usj.edu.lb

How to cite this article: Manhal W, Yared G, Tahtouh R, et al. Poor Biointegration of Porcine Acellular Dermal Matrix Associated with Unfavorable Gingival Healing: A Report of Three Cases. J Contemp Dent Pract 2021;22(8):951-958.

Source of support: Nil

Conflict of interest: None

(0) The Author(s). 2021 Open Access This article is distributed under the terms of the Creative Commons Attribution 4.0 International License (https://creativecommons. org/licenses/by-nc/4.0/), which permits unrestricted use, distribution, and non-commercial reproduction in any medium, provided you give appropriate credit to the original author(s) and the source, provide a link to the Creative Commons license, and indicate if changes were made. The Creative Commons Public Domain Dedication waiver (http://creativecommons.org/publicdomain/zero/1.0/) applies to the data made available in this article, unless otherwise stated. 
alloplastic substitutes, which were proven to be a safe solution despite having lower success rates than autogenous grafts. ${ }^{10}$ Moreover, the use of ADM allografts may be associated with ethical concerns and the potential risk of disease transmission. Thus, as an alternative, xenogeneic acellularized materials were introduced. ${ }^{11}$ Several clinical and preclinical studies reported their successful use for root coverage and soft tissue thickening procedures. ${ }^{12,13}$

Because of its nonvital structure, ADM acts as a threedimensional scaffold that depends on recipient site cells and blood vessels to achieve reorganization, causing a slower incorporation that could ultimately result in structural and functional impairment. This original graft matrix then degrades through new connective tissue production and eventually becomes completely replaced by host tissues. ${ }^{14}$ However, the efficiency of cell incorporation throughout the matrix is still under investigation as previous studies showed that PADM are only partially colonized in vitro by gingival fibroblasts. Thus, cells are preferentially located on the surface and unable to migrate from the periphery of the matrix inward. ${ }^{15,16}$

As for autogenous grafts, ADM was proposed as an alternative for ridge augmentation, and soft tissue thickening ${ }^{17}$ to correct aesthetic and functional problems. ${ }^{18}$

In the present study we report adverse outcomes observed in three patients grafted with pADM to treat soft tissue deficiencies around teeth and implants. Due to these unsatisfactory clinical results, it became necessary to understand the dynamics of pADM in situ biointegration. For this purpose, it was possible to observe histologically the biointegration of the current grafted pADM. Furthermore, in order to elucidate the histologically observed poor pADM biointegration, ultrastructural analyses, and in vitro studies using human gingival fibroblast cultures were also performed.

\section{Case Description}

This study involved three patients showing soft tissue deficiencies who underwent soft tissue grafting surgery (at Saint-Joseph University Dental Clinics), using pADM as an alternative to connective tissue autogenous graft. Two out of three (Patients \#1 and \#2) presented gingival recessions resulting in tooth sensitivity and unaesthetic aspect while the third one (Patient \#3) had a buccal horizontal bone defect impairing both the esthetic and functional results of the future implant site. We had the opportunity to harvest biopsies in two out of the three patients (Patients \#2 and \#3) without any modification of the surgical procedure.

\section{Patient 1}

The first patient was a 37-year-old male with a 2-mm gingival recession (Class 1 of Miller) on the first upper left premolar (Fig. 1A). In order to harmonize the gingival smile line and reduce hypersensitivity, a buccal recession on tooth 24 was grafted with a pADM then covered with a partial thickness coronally repositioned
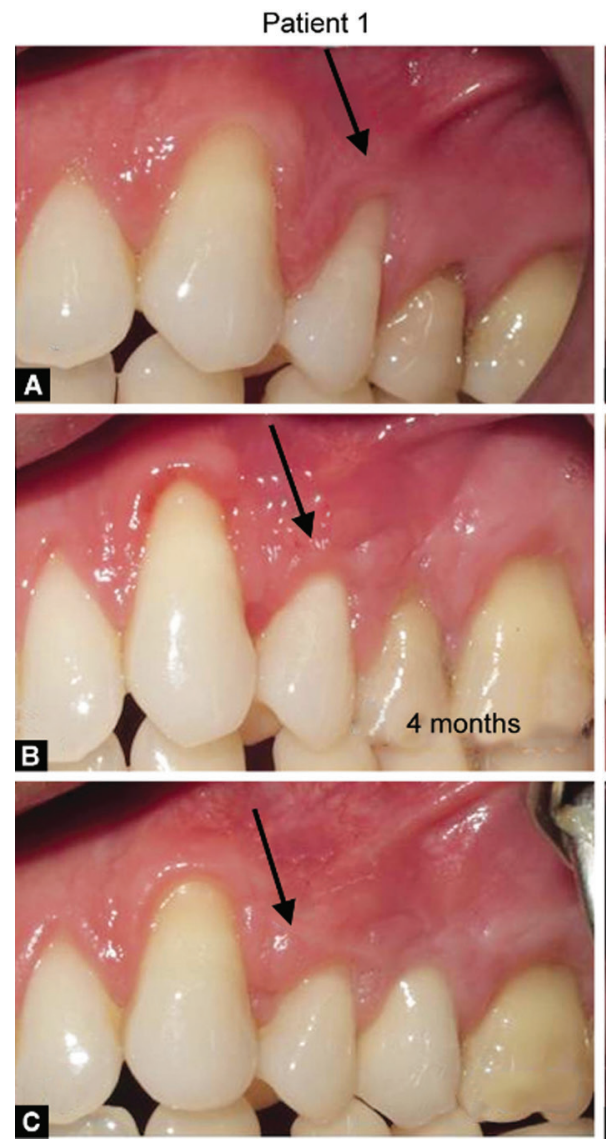

Patient 2
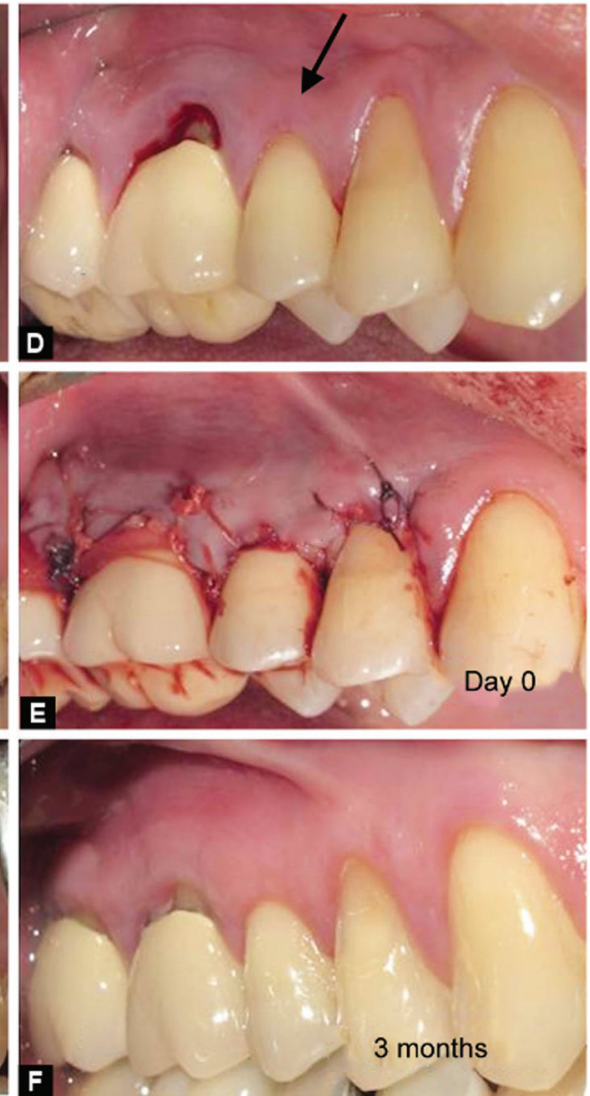

Patient 3
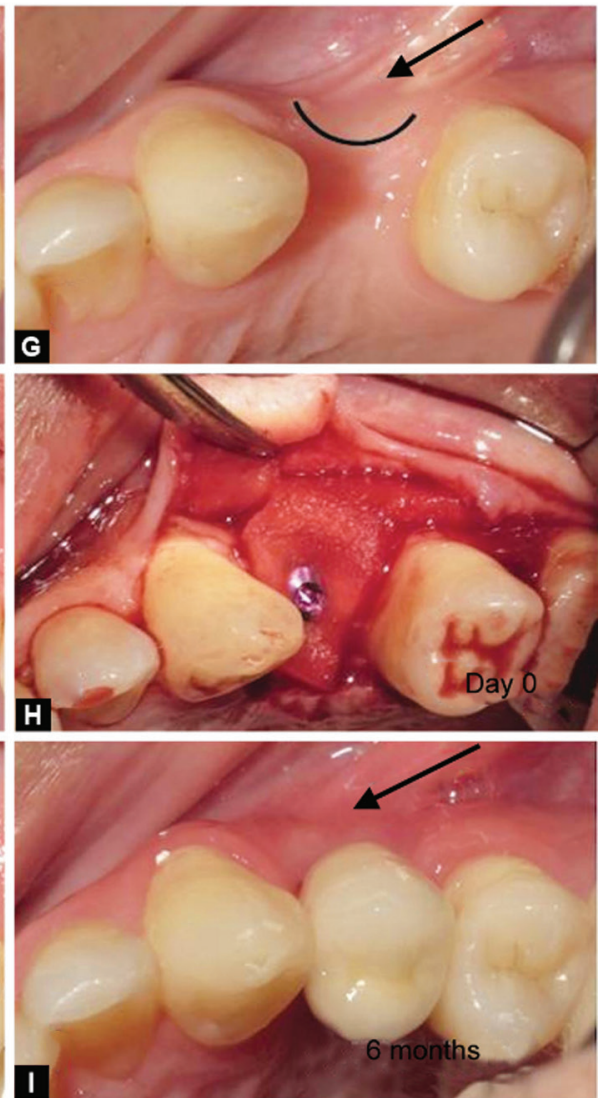

Figs 1 A to I: Clinical observations: Patients 1 (A to C), 2 ( $D$ to F), 3 (G to I). (A) Recession on 24; (B) Redness persists, 4 months following surgery; (C) At 1-year, redness persistence; (D) Gingival recession on 14, 15; (E) Day 0, partial-thickness tunnel flap; (F) Three months, total root coverage; (G) Restoration site; (H) pADM designed according to the buccal defect; (I) Six months after prosthetic restoration, unsuitable redness was still observed 
flap. ${ }^{19}$ Following total root debridement, the membrane was thoroughly hydrated in a saline buffer then reshaped according to the grafted site and positioned apically to the cementoenamel junction and stabilized with absorbable sutures. Four months following the PADM grafting procedure, a clinical examination of the surgical site revealed poor healing with persistence of redness corresponding to gingival inflammation that was painless yet firm upon palpation (Fig. 1B). At 1-year post-op, the color of the grafted area was still discordant with its surrounding gingiva, and the gum recession was reduced to $0.5 \mathrm{~mm}$ while the keratinized tissue thickness was $2-3 \mathrm{~mm}$. Furthermore, palpation confirmed the persistence of a firm mass at the grafted site (Fig. 1C).

\section{Patient 2}

The second patient was a 33-year-old male complaining from tooth hypersensitivity caused by a gingival recession (Class 1 Miller) on the two upper right premolars (14 and 15) with no carious cervical lesions (Fig. 1D). The recessions were treated using a tunnel technique. Following the preparation of a partial-thickness flap mobilizing the marginal gingiva, a mucogingival tunnel was prepared into which the pADM membrane was introduced and stabilized by sutures (Fig. 1E). ${ }^{20}$ At 3 months, a noticeable increase in the attached gingiva was observed with total root coverage of the second right premolar (Fig. 1F). However, the grafted site clinically showed a normal-looking attached gingiva in harmony with its surroundings. At 27 weeks, concomitantly with a homolateral wisdom tooth removal next to the grafted area, a biopsy was carried out within the grafted site for histological analysis of the pADM biointegration, with the patient's consent without modifying the original surgical procedure.

\section{Patient 3}

The third patient was a 35-year-old male who came in for the replacement of his edentulous first left upper premolar. Clinical examination revealed a thin biotype associated with a reduced amount of keratinized gingiva in both height and thickness (Fig. 1G). No periodontal pockets were revealed after probing. The treatment of choice was implant rehabilitation for conservative and functional reasons. The presence of a horizontal bone defect at the future implant site had to be treated to enhance the buccal contour. Then a PADM was hydrated in a saline buffer, designed and secured in place according to the buccal defect following a full thickness flap (Fig. 1H). Eleven weeks after surgery, the procedure seemed to yield acceptable clinical results since the postextraction horizontal bone defect appeared to be compensated. However, gingival redness was still present at the surgical site. At 11 weeks postgrafting, a gingival biopsy was collected at the grafted site for histological analysis, while uncovering the implant during the second stage surgery, as it involved the removal of the keratinized tissue (1-2 mm) covering the cover screw and the healing abutment placement. ${ }^{21} \mathrm{~A}$ prosthetic rehabilitation was performed 30 days later. Six months after the prosthesis placement, gum covering the grafted site was marked by a persistent redness that could be considered aesthetically unsatisfactory (Fig. 1I).

\section{Histological Observations of Gingival Biopsies from the Second and Third Case}

Biopsies collected during the surgical procedures ( 27 and 11 weeks after surgery, respectively) were routinely prepared for histological analysis on paraffin $5 \mu \mathrm{m}$ sections. ${ }^{22,23}$

\section{Patient 2}

Hematoxylin-eosin staining showed numerous elongated fibroblastic cells and round-shaped inflammatory cells invading fibrous tissue in the grafted site (Figs $2 A$ and $B$ magnifications $\times 26$ and $\times 52$, respectively). Verhoff-Van Gieson staining revealed gingival epithelium, connective tissue, and alveolar mucosa (Fig. 2C, $\times 26$ ). In the grafted area next to the alveolar bone, the deep connective tissue showed a red stained fibrillar material, indicating a very dense extracellular matrix. Higher magnification highlighted collagen and cell abundance consistent with a fibrotic process, as well as some shortened elastic fibers (Fig. 2D, arrows, $\times 52$ ).

\section{Patient 3}

The histological examination of Hematoxylin-eosin-stained gingival sections revealed a well-defined border between pADM and the inflammatory gingival connective tissue (Fig. $3 A, \times 26$ ). The blood vessels originating from both the flap and periosteum only invade the peripheral portion of PADM, indicating marginal neo-angiogenesis. At higher magnification, Verhoeff-Van Gieson staining highlighted the polymorphous inflammatory infiltrate including macrophages, lymphocytes, plasma cells, and foreign giant cell granulomas around lamellar structures (Figs 3B and C, $\times 52$ ). Additionally, numerous fibroblast-like elongated cells were present in the ulceration, as well as newly formed collagen fibers. Periodic acid-Schiff red-stained lamellar structures (Figs 3D, $\times 26$ and $3 \mathrm{E}, \times 52$ ) surrounded by foreign giant cell granulomas (Fig. 3F, $\times 52$ ) could correspond to the pADM residues.

\section{Ultrastructural and Histological Characterizations of pADM Prior to Implantation or Cell Culture}

According to the manufacturer, the pADM used in the present study (Mucoderm ${ }^{\oplus}$ Botiss Biomaterials, Berlin, Germany) undergo a process that allows the decellularization and elimination of any potentially antigenic determinant, making them safe to use for humans. Scanning electron microscopy showed that the pADM surface displayed a very dense network including shallow pores, which corresponded to dermis depressions resulting from the loss of epidermal appendages during the preparative process (Figs $4 \mathrm{~A}$, $\times 50$ and $4 B, \times 100$ ). At higher magnification, pADM appeared to be composed of several dense layers of interconnected collagen fiber aggregates (Figs $4 C, \times 600$ and 4D, $\times 1,000$ ); some of them presenting characteristic periodic striations (Fig. 4D). Under polarized light microscopy and after picrosirius red staining, pADM displayed large collagen bundles (diameter $>50 \mu \mathrm{m}$ ), with a few interfibrillar spaces (Fig. 4E, $\times 52$ ) as compared to normal human gingiva. ${ }^{24} \mathrm{At}$ high magnification, the pADM collagen network seemed to be constituted of aggregated fibers forming large and abnormal wavy bundles (Fig. 4F, $\times 126$ ).

\section{Morphology and Distribution of Cultured Gingival Fibroblasts on PADM}

Human gingival fibroblasts were obtained from gingival biopsies taken from six patients (18-30 years old) free of pathologies after obtaining their written informed consent in accordance with the principles outlined in the Declaration of Helsinki. Gingival fibroblasts were then cultivated on a piece of PADM in standard conditions. 10, 15, and 25 days after cell seeding, cultured pADM were prepared for standard histological procedure and specifically stained. The Hematoxylin-eosin staining showed an early onset of adherent fibroblasts on the pADM surface (Figs 5A to D). During 

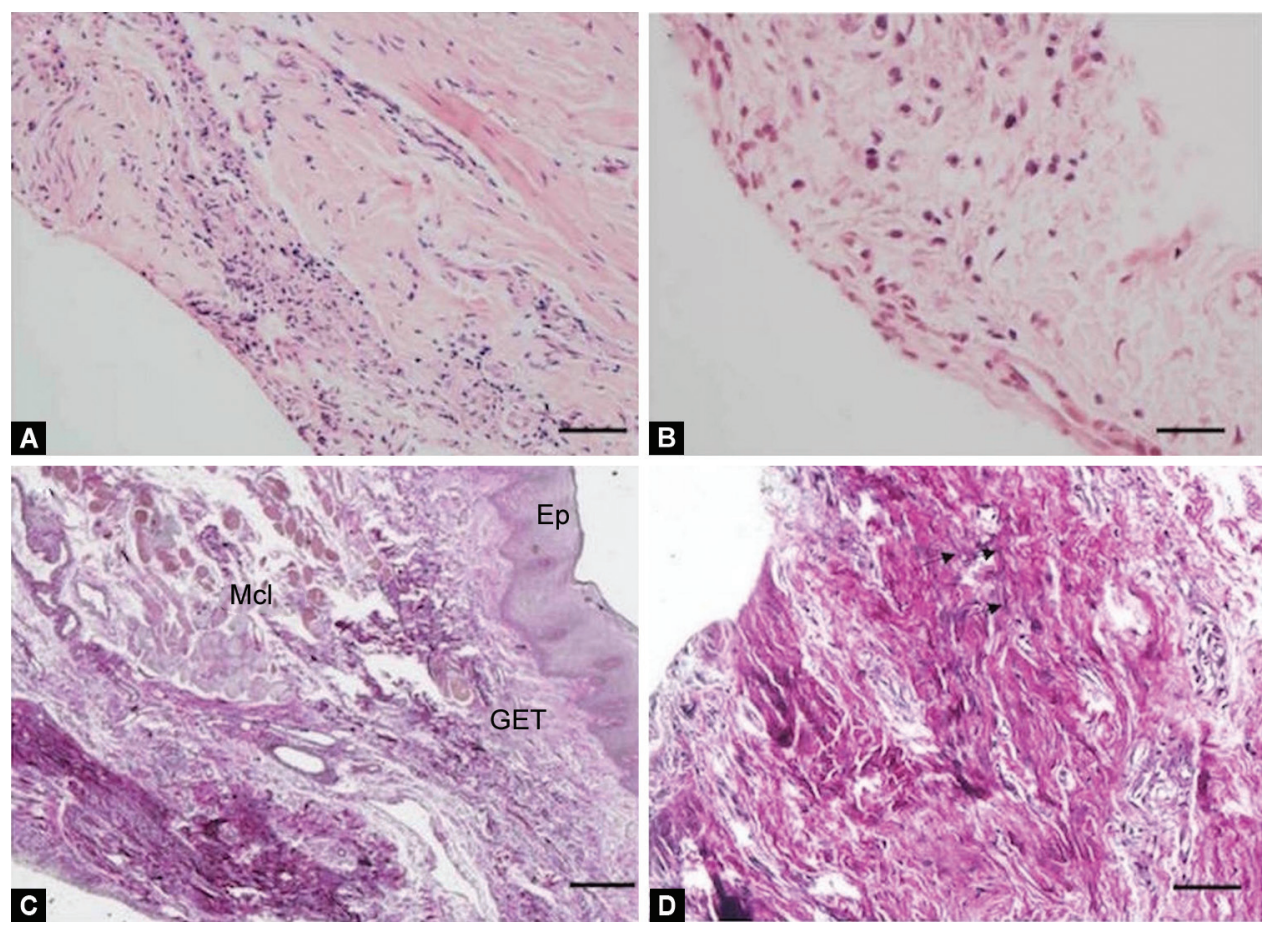

Figs 2A to D: Patient 2 grafted gingival site. (A) Inflammatory infiltrate; (B) Round inflammatory cells and other more elongated fibroblastic cells; (C) Very dense collagen network with some shortened elastic fibers; (D) Fibrotic area. (A, B) Hematoxylin eosin staining. (C, D) Verhoeff-Van Gieson staining. Ep. Epithelium, GCT. gingival connective tissue, mcl. muscle tissue. Scale bars: $(A, C)=100 \mu m,(B, D)=50 \mu m$
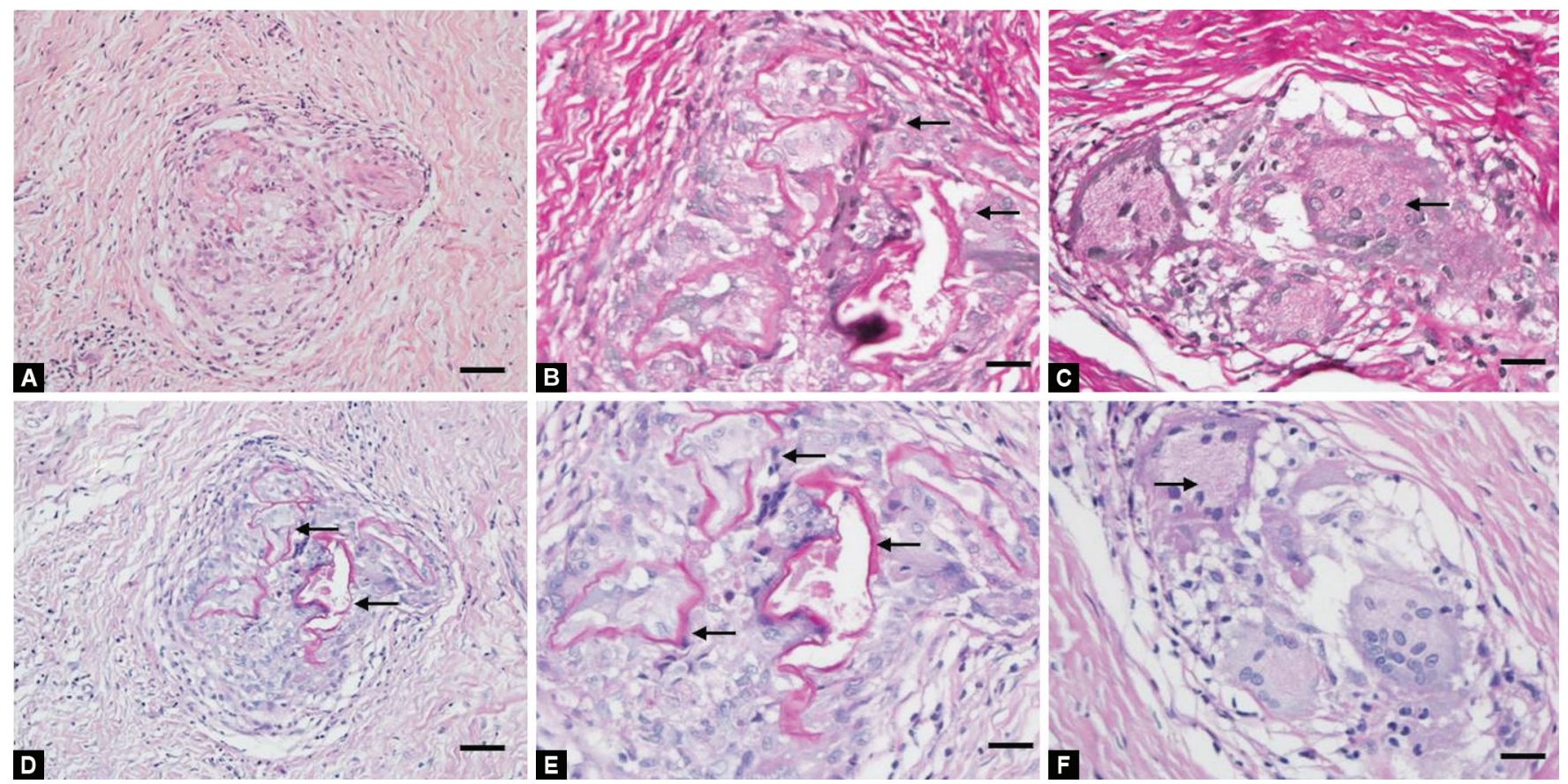

Figs 3 A to F: Patient 3 grafted gingival site. (A) Connective tissue surrounding ulceration; (B) Ulceration showing foreign giant cell granulomas and lamellar structure attributed to pADM; (C) Giant foreign body type cells (arrows); (D, E) Ulceration containing the rest of pADM (red lamellar structures, arrows); (F) Giant foreign type cells (arrow); (A) Hematoxylin eosin staining; (B, C) Verhoeff-Van Gieson staining; (D to F) Periodic acidSchiff staining. Scale bars: $(A, D)=100 \mu \mathrm{m},(B, C, E, F)=25 \mu \mathrm{m}$

culture times, no deep pADM colonization by any of the used gingival fibroblast strains $(n=6)$ was observed except when cells migrated in the collagen matrix fracture areas while remaining at the surface (Figs $5 A$ and $B, \times 26$ ). 

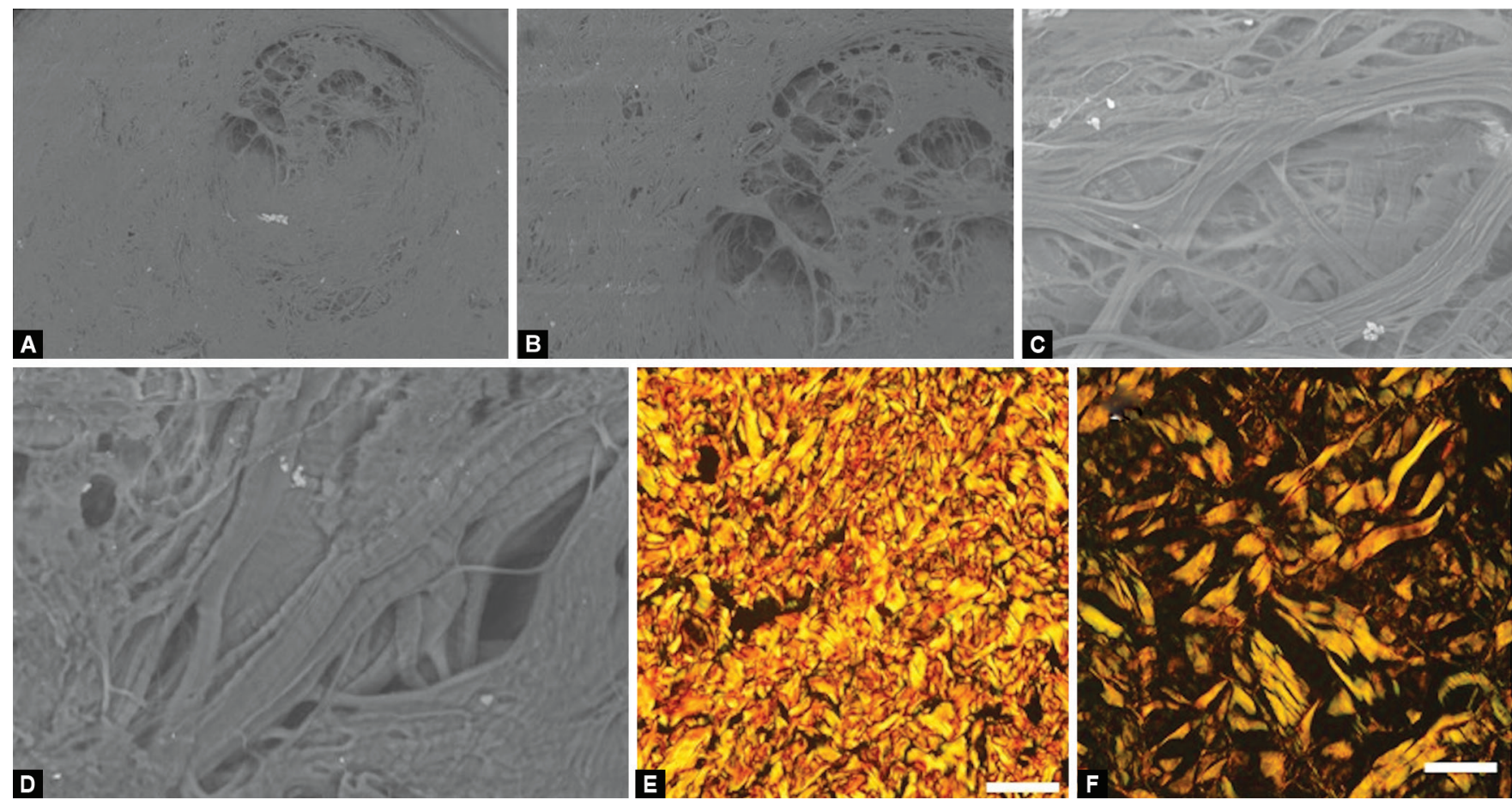

Figs 4A to F: pADM collagen network: scanning electron micrograph and picrosirius red staining. (A) Global ultrastructure of the pADM surface; (B) Surface depression in pADM: remains of skin appendage; (C) pADM dense collagen network; (D) pADM collagen bundles with periodic banding pattern; (E, F) Picrosirius red staining under polarized light. Scale bars: $(E)=200 \mu \mathrm{m},(F)=100 \mu \mathrm{m}$

Ten days after seeding, the adherent cells were found unevenly distributed on the PADM surfaces and displayed a polygonal or elongated shape with round nuclei. At 15 days, some isolated pADM areas were occupied by confluent elongated fibroblasts (Fig. 5C, $\times 26$ ). After 25 days, fibroblasts formed a continuous layer over the pADM surface. Furthermore, some sites were occupied by fibroblast multilayers suggesting a preferential peripheral cell tridimensional layout and intercellular matrix synthesis (Fig. 5D, ×52).

Collagen matrix remodeling of cultured pADM was evidenced by picrosirius red staining. Collagen material is stained in red under transmitted light and the fibrillar nature of the collagen network is revealed by its birefringence under polarized microscopy. In longterm cultures, the pADM portion in contact with cells is substantially degraded, as highlighted by picrosirius red staining (Figs $6 \mathrm{~A} \times 26$ and $6 \mathrm{~B}, \times 52$ ). At high magnification, the collagen hydrolysis was clearly observed at the PADM surface, while its inner part remained preserved (Fig. $6 C, \times 104$ ). Thus, under polarized light, the loss of birefringence revealed transformation of fibrillar collagen into gelatin, which demonstrated that the pADM peripheral area underwent time-dependent collagen hydrolysis over the course of the culture (Figs $6 \mathrm{D}$ to $6 \mathrm{~F}, \times 26$ ).

\section{Discussion}

This study focuses on pADM bio integration as a gingival substitute in periodontal surgery mainly used for soft tissue augmentation. ${ }^{10}$ In fact, several studies reported the effectiveness of pADM and other soft tissue substitutes in improving the periodontal condition, emphasizing the favorable clinical aspect of volume augmentation, ${ }^{5-8}$ however, these procedures require a better knowledge of wound healing, so that soft tissue substitutes are regarded as a completely viable option for gingival augmentation. ${ }^{25,26}$ Therefore, it seems necessary to understand the intimate mechanism of pADM biointegration in the rare situations associated with unfavorable gingival healing. ${ }^{27}$ Thus, ideally, after in vivo grafting, $\mathrm{pADM}$ should be finally replaced by a newly formed gingival connective tissue. ${ }^{7}$ In order to do so, the exogenous matrix must be absorbed and invaded by cells (gingival fibroblasts, endothelial cells, and inflammatory cells) and blood vessels that allow tissue reformation. ${ }^{28}$ Understanding the mechanisms leading to the biointegration of the exogenous matrix was crucial to fully interpret the poor clinical results observed in the current case reports. Thus, several months after the grafting procedure of both the first and third patients presented in this study were characterized by a persistent gingival redness and a partial loss of the reconstructed gingival volume. This is in accordance with a previous study showing a slower and incomplete healing when using pADM compared to autogenous CTGs. ${ }^{29}$

This lasting connective tissue redness could be associated with chronic inflammation even though soft tissue substitute procedures are reported to incur a lower likelihood for postoperative pain or bleeding than autogenous procedures. ${ }^{8,29}$ Additionally, deep palpation revealed the presence of a denser than normal tissue that could be either nonresorbed pADM or newly formed fibrous tissue.

Thus, the histological examination of the third patient biopsy taken 11 weeks after surgery showed a persistent granulation tissue and inflammatory infiltrate within the grafted site. The chronic inflammatory reaction was marked by the presence of giant foreign body cells surrounding pADM as already reported with the same membrane after subcutaneous grafting in mice. ${ }^{30}$ Curiously, even 


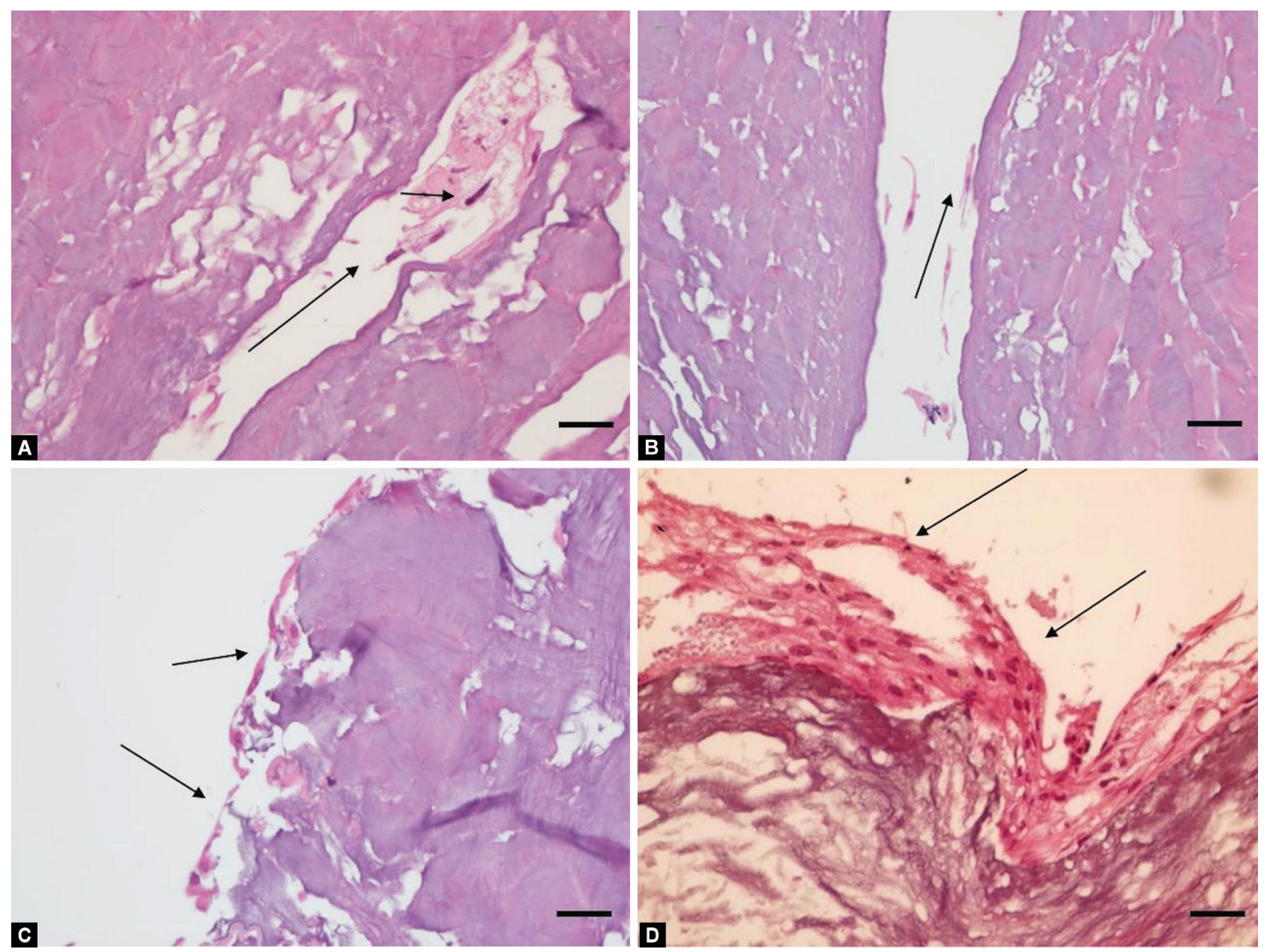

Figs $5 A$ to $D$ : Cultures of human normal gingival fibroblasts on pADM. (A, B) Gingival fibroblasts on the pADM surface after 10 days; (C) Gingival fibroblasts on the pADM surface after 15 days; (D) Gingival fibroblasts form multilayers on the pADM surface after 25 days. (A to D) Hematoxylin eosin staining. Scale bars: (A to $C)=50 \mu \mathrm{m} ;(D)=25 \mu \mathrm{m}$

though the second patient yielded acceptable clinical results, histological sections showed that 27 weeks after grafting, a clear fibrosis and a considerable inflammatory infiltration were associated with the pADM. ${ }^{31}$

Besides, this fibrous tissue was poorly vascularized and contained thick collagen fibers isolating the implanted xenogenic substitute from surrounding connective tissue, and that could be regarded as a later stage than it had been observed for the third patient. Aggregation and fusion of macrophages leading to the formation of a multinucleated foreign body reaction and associated with substantial fibrosis are one of the hallmarks of the foreign body response. It was obviously regarded as incompatible with successful tissue-engineering outcomes. ${ }^{32}$ This type of reaction usually occurs when foreign bodies cannot be eliminated by phagocytosis, enzymatic or inflammatory oxidative processes and need to be isolated. ${ }^{33}$ Interestingly, lamellar structures highlighted by Periodic acid-Schiff reaction could show that some glycosylated materials remained in grafted PADM and explain, in part, the observed immune and inflammatory reactions. The bad biointegration could also be due to the inability of the tissue reaction to both achieve the pADM resorption and to allow both gingival fibroblasts and newly formed blood vessel invasions. As previously shown adhesion, spreading, and proliferation of human gingival fibroblasts on the pADM surface demonstrated that specific and various collagen adhesion sites were still preserved and accessible for cell surface integrins. ${ }^{34}$ However, the present study showed that, after 25 days of culture, the human gingival fibroblasts were still unable to invade the inner part of the pADM. In fact, the histological and ultrastructural studies performed before culture showed that the pADM collagen architecture differed from the normal gingival collagen network. Indeed, the histological appearance of normal gingival and dermal connective tissues, after picrosirius red staining, ${ }^{35}$ was characterized by a 10-20 $\mu \mathrm{m}$ range of fibrillar collagen fiber diameters with numerous and uniformly distributed interfibrillar spaces. ${ }^{36}$ The abnormal appearance of pADM was characterized by very large collagen fibers (diameter $>50 \mu \mathrm{m}$ ) and a marked decrease of interfibrillar spaces. Furthermore, under scanning electron microscopy, the pADM displays a very thick and dense fibrous network without apparent interconnected porosity. Yet, other culture models showed that, after seeding, fibroblasts were able to deeply invade dense reconstructed collagen matrices without apparent pores. In these models, deep invasion and migration throughout the matrices were an MMP-dependent process involving cooperative activities 

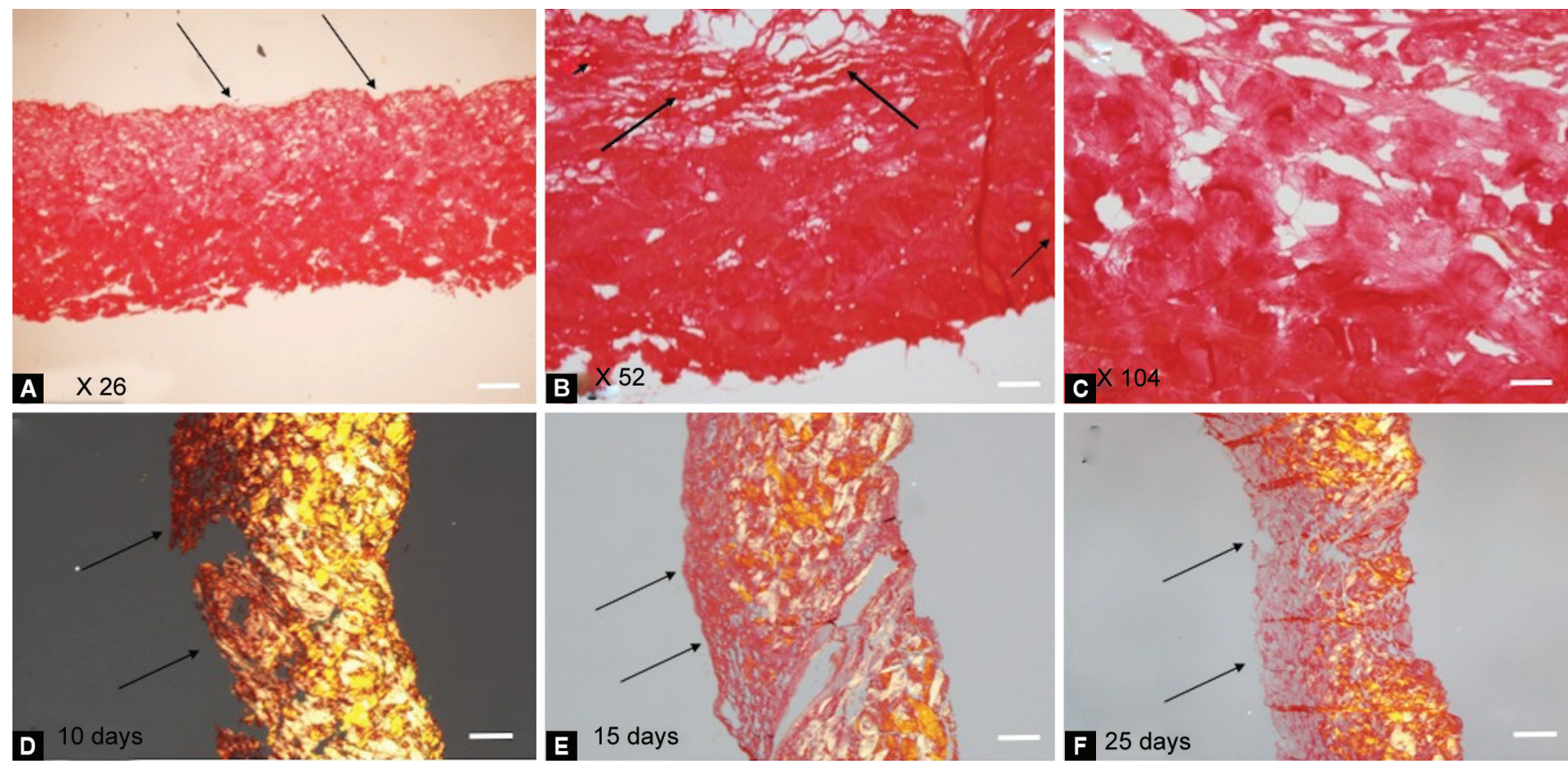

Figs $6 \mathrm{~A}$ to F: Hydrolysis of cultured pADM collagen network by human normal gingival fibroblasts (picrosirius red staining). (A to C) Collagen degradation from the cultured surface (arrows) to the depth of pADM after 25 days (transmitted light); (D to F) Fibrillar collagen gradient of degradation characterized by birefringence loss (arrows) during culture course. $(D)=10$ days, $(E)=15$ days, $(F)=25$ days (polarized light). Scale bars: $(A, D, E, F)=100 \mu m,(B)=50 \mu m,(C)=25 \mu \mathrm{m}$

of both collagenases (MMP-1) and gelatinases (MMP-2). ${ }^{37}$ Under polarized light, picrosirius red staining showed a progressive loss of collagen birefringence from the surface to the internal part of the cultured PADM, evidencing transformation of fibrillar collagen network into gelatin. As expected, gradient degradation of the fibrillar collagen network may be due to collagenase secretion by proliferating gingival fibroblasts on the pADM surface. Thus, it can be clearly hypothesized that the resulting gelatin matrix was resistant to gelatinolytic activities, which prevents in-depth cell migration into the pADM membrane. Consequently, the proteolytic resistance of the membrane could be due to the formation of tight crosslinks between the collagen bundles during the manufacturing process and could explain the inability of surrounding tissue to integrate pADM. In addition, the highly dense pADM collagen network seems to limit in vitro cell colonization and both in vitro and in vivo tissue remodeling. Thus, inconsistent clinical results can be explained by a macromolecular pADM structure observably different from the physiological gingiva, despite their assumed common macromolecular composition.

\section{Conclusion}

The observed fibrotic process and the persistence of giant multinucleated foreign body cells evidence the poor ADM integration and the sustained inflammation of the grafted site. The usefulness of this kind of membrane is undeniable for soft tissue augmentation. However, its manufacturing process requires some improvements in order to achieve in vivo remodeling, and consequently better biointegration.

\section{Ethics}

This research project "Poor biointegration of porcine ADM associated with unfavorable gingival healing: a report of three cases," has been validated by the Ethics Committee of Saint Joseph University (ref: USJ-2013-17) in accordance with the Declaration of Helsinki.

\section{ACKnOWLedgments}

Authors are grateful for the financial support received from SaintJoseph University of Beirut, Sorbonne Paris Nord University, and School of Biological Industry (EBI), for this research, authorship, and/or publication of this article.

\section{ORCID}

Wassim Manhal 이 https://orcid.org/0000-0002-2062-8343

Ronald Younes (ㄷ) https://orcid.org/0000-0002-4001-9879

\section{References}

1. Talwar BS. A focus on soft tissue in dental implantology. J Indian Prosthodont Soc 2012;12(3):137-142. DOI: 10.1007/s13191-012-0133-x.

2. Mounssif I, Stefanini M, Mazzotti $C$, et al. Esthetic evaluation and patient-centered outcomes in root-coverage procedures. Periodontol 2000 2018;77(1):19-53. DOI: 10.1111/prd.12216.

3. Poli PP, Beretta M, Grossi GB, et al. Risk indicators related to periimplant disease: an observational retrospective cohort study. J Periodontal Implant Sci 2016;46(4):266-276. DOI: 10.5051/ jpis.2016.46.4.266.

4. Schrott AR, Jimenez M, Hwang JW, et al. Five-year evaluation of the influence of keratinized mucosa on peri-implant soft-tissue health and stability around implants supporting full-arch mandibular fixed prostheses. Clin Oral Implants Res 2009;20(10):1170-1177. DOI: 10.1111/j.1600-0501.2009.01795.x.

5. Lorenzo R, García V, Orsini M, et al. Clinical efficacy of a xenogeneic collagen matrix in augmenting keratinized mucosa around implants: a randomized controlled prospective clinical trial. Clin Oral Implants Res 2011;23(3):316-324. DOI: 10.1111/j.1600-0501.2011.02260.x. 
6. Griffin TJ, Cheung WS, Hirayama H. Hard and soft tissue augmentation in implant therapy using acellular dermal matrix. Int J Periodontics Restorative Dent 2004;24(4):352-361. DOI: 10.11607/prd.00.0591.

7. Cummings LC, Kaldahl WB, Allen EP. Histologic evaluation of autogenous connective tissue and acellular dermal matrix grafts in humans. J Periodontol 2005;76(2):178-186. DOI: 10.1902/ jop.2005.76.2.178.

8. Griffin TJ, Cheung WS, Zavras Al, et al. Postoperative complications following gingival augmentation procedures. J Periodontol 2006;77(12):2070-2079. DOI: 10.1902/jop.2006.050296.

9. Harris RJ. A short-term and long-term comparison of root coverage with an acellular dermal matrix and a subepithelial graft.J Periodontol 2004;75(5):734-743. DOI: 10.1902/jop.2004.75.5.734.

10. Caballé-Serrano J, Zhang S, Ferrantino $L$, et al. Tissue response to a porous collagen matrix used for soft tissue augmentation. Materials (Basel) 2019;12(22). DOI: 10.3390/ma12223721.

11. Pabst AM, Happe A, Callaway A, et al. In vitro and in vivo characterization of porcine acellular dermal matrix for gingival augmentation procedures. J Periodontal Res 2014;49(3):371-381. DOI: 10.1111/jre.12115.

12. Schmitt CM, Schlegel KA, Gammel L, et al. Gingiva thickening with a porcine collagen matrix in a preclinical dog model: Histological outcomes. J Clin Periodontol 2019;46(12):1273-1281. DOI: 10.1111/ jcpe.1319.

13. Thoma DS, Naenni N, Figuero E, et al. Effects of soft tissue augmentation procedures on peri-implant health or disease: a systematic review and meta-analysis. Clin Oral Implants Res 2018;29(S15):32-49. DOI: 10.1111/clr.13114.

14. Wei $P-C$, Laurell L, Lingen MW, et al. Acellular dermal matrix allografts to achieve increased attached gingiva. Part 2. A histological comparative study. J Periodontol 2002;73(3):257-265. DOI: 10.1902/ jop.2002.73.3.257.

15. Rodrigues AZ, Oliveira PT de, Novaes Jr AB, et al. Evaluation of in vitro human gingival fibroblast seeding on acellular dermal matrix. Braz Dent J 2010;21(3):179-189. DOI: 10.1590/s0103-64402010000300001.

16. Rothamel D, Schwarz F, Sager M, et al. Biodegradation of differently cross-linked collagen membranes: an experimental study in the rat. Clin Oral Implants Res 2005;16(3):369-378. DOI: 10.1111/j.16000501.2005.01108.x.

17. Puisys A, Žukauskas $S$, Kubilius $R$, et al. Bone augmentation and simultaneous soft tissue thickening with collagen tissue matrix derivate membrane in an aesthetic area. A case report. Stomatologija 2017;19(2):64-68. PMID: 29243686.

18. Meltzer JA. Edentulous area tissue graft correction of an esthetic defect: a case report. J Periodontol 1979;50(6):320-322. DOI: 10.1902/ jop.1979.50.6.320.

19. de Sanctis M, Baldini N, Goracci C, et al. Coronally advanced flap associated with a CTG for the treatment of multiple recession defects in mandibular posterior teeth. Int J Periodontics Restorative Dent 2011;31(6):623-630. DOI: 10.11607/prd.00.1017.

20. Allen AL. Use of the supraperiosteal envelope in soft tissue grafting for root coverage. II. Clinical results. Int J Periodontics Restorative Dent 1994;14(4):302-315. DOI: 10.11607/prd.00.0091.

21. Suchetha A, Phadke PV, Sapna N, et al. Optimising esthetics in second stage dental implant surgery: periodontist's ingenuity. J Dent Implants 2014;4(2):170. DOI: 10.4103/0974-6781.140898.

22. Matenaers C, Popper B, Rieger A. Practicable methods for histological section thickness measurement in quantitative stereological analyses. PLoS One 2018;13(2):e0192879. DOI: 10.1371/journal. pone.0192879.

23. Kawasaki $\mathrm{H}$. The NanoSuit method: a novel histological approach for examining paraffin sections in a nondestructive manner by correlative light and electron microscopy. Lab Invest 2020;100: 161-173. DOI: 10.1038/s41374-019-0309-7.

24. Younes R, Ghorra C, Khalife S, et al. Pertinent cell population to characterize periodontal disease. Tissue Cell 2009;41(2):141-150. DOI: 10.1016/j.tice.2008.09.003.

25. Agarwal C, Tarun Kumar AB, Mehta DS. Comparative evaluation of free gingival graft and AlloDerm ${ }^{\circledast}$ in enhancing the width of attached gingival: a clinical study. Contemp Clin Dent 2015;6(4):483-488. DOI: 10.4103/0976-237X.169838.

26. Toledano-Osorio M, Carrasco-Carmona A, Vallecillo C, et al. State of the art on biomaterials for soft tissue augmentation in the oral cavity. Part I: natural polymers-based biomaterials manuel. Polymers 2020;12(8):1850. DOI: 10.3390/polym12081850.

27. Rothamel D, Benner M, Fienitz T, et al. Biodegradation pattern and tissue integration of native and cross-linked porcine collagen soft tissue augmentation matrices - an experimental study in the rat. Head Face 2014;10:10. DOI: 10.1186/1746-160X-10-10.

28. Côrtes ADQ, Martins AG, Nociti FH, et al. Coronally positioned flap with or without acellular dermal matrix graft in the treatment of Class I gingival recessions: a randomized controlled clinical study. J Periodontol 2004;75(8):1137-1144. DOI: 10.1902/jop.2004.75.8.1137.

29. Paolantonio M, Dolci M, Esposito $P$, et al. Subpedicle acellular dermal matrix graft and autogenous connective tissue graft in the treatment of gingival recessions: a comparative 1-year clinical study. J Periodontol 2002;73(11):1299-1307. DOI: 10.1902/jop.2002.73.11.1299.

30. Barbeck M, Lorenz J, Kubesch A, et al. Porcine dermis-derived collagen membranes induce implantation bed vascularization via multinucleated giant cells: a physiological reaction? J Oral Implantol 2015;41(6):e238-e251. DOI: 10.1563/aaid-joi-D-14-00274.

31. Zuo Y, Lu S. Dermis, acellular dermal matrix, and fibroblasts from different layers of pig skin exhibit different profibrotic characteristics: evidence from in vivo study. Oncotarget 2017;8(14):23613-23627. DOI: 10.18632/oncotarget.15389.

32. Anderson JM, Rodriguez A, Chang DT. Foreign body reaction to biomaterials. Semin Immunol 2008;20(2):86-100. DOI: 10.1016/j. smim.2007.11.004.

33. Harris RJ. Root coverage with a connective tissue with partial thickness double pedicle graft and an acellular dermal matrix graft: a clinical and histological evaluation of a case report. J Periodontol 1998;69(11):1305-1311. DOI: 10.1902/jop.1998.69.11.1305.

34. Heino J. The collagen receptor integrins have distinct ligand recognition and signaling functions. Matrix Biol 2000;19(4):319-323. DOI: 10.1016/s0945-053x(00)00076-7.

35. Lattouf $R$, Younes $R$, Lutomski $D$, et al. Picrosirius red staining: a useful tool to appraise collagen networks in normal and pathological tissues. J Histochem Cytochem 2014;62(10):751-758. DOI: $10.1369 / 0022155414545787$.

36. Senni K, Godeau G. Aspect histologique et macromoléculaire de la gencive saine. In: Bouchard P, editor. Parodontologie et Dentisterie Implantaire. Paris: Lavoisier Médecine; 2014. p. 13-18.

37. Helary C, Abed A, Mosser G, et al. Evaluation of dense collagen matrices as medicated wound dressing for the treatment of cutaneous chronic wounds. Biomater Sci 2015;3(2):373-382. DOI: 10.1039/c4bm00370e. 